\title{
MORFOLOGIA DEL CIRCO DE TUCARROYA (Macizo de Monte Perdido, Pirineo Aragonés)
}

\author{
Pedro M. Nicolás-Martínez
}

\begin{abstract}
SUMMARY
The author analizes one of the most spectacular examples of glacier morphology that can be found in the Spanish Pyrenees. After exposing the most important structural aspects, the karstic shaping, the glacial and periglacial processes will be analized succesively. The present study will be mainly centered in the description and explanation of the forms originated by pleistocenic glaciers and also in the new and recent glaciers. We quoted several moraines from a historic epoque and we point out some basic characteristics of recent residual glaciers.
\end{abstract}

El área objeto de trabajo es aquella que Schrader bautizó como "Plateau de Mont Perdu" y que nosotros hemos denominado Circo de Tucarroya. Los límites de este alto cuenco están constituidos por dos importantes barreras montañosas, una al N-E y otra al S-SO. Al NO. se cierra el Circo por un alto collado, que muestra en su vertiente opuesta las magníficas proporciones del circo francés de $\mathrm{Ga}$ varnie.

Aguas abajo, el Circo se corta bruscamente, en la cota 2.500, en un escarpe en forma arqueada, que da paso a una fuerte pendiente de unos $800 \mathrm{mts}$. ocupada por gleras, pastizales y paredes rocosas, que unen la zona de Tucarroya con la que conocemos por el nombre de Valle de Pineta.

El cordal de cumbres que limitan esta alta plataforma alcanza su máxima altitud en el Monte Perdido (la más meridional de ellas),

1. Según el topónimo usado por Schrader en la reproducción de su mapa de Monte Perdido en la página 33 de Pyrénées, tomo I. 
con $3.352 \mathrm{mts}$. de altura. El punto más bajo de toda esta larga cresta se sitúa en los $2.680 \mathrm{~m}$., aproximadamente, de la Brecha de Tucarroya.

La atención científica a esta zona surge ya a finales del siglo XVIII y a principios del XIX con las obras de Ramond de Carbonniéres, en las que, a una romántica visión del paisaje de las altas montañas, añade ya unos claros deseos de conocimiento. Esta actitud está en la misma línea que la toma da pocos años antes en los Alpes por los naturalistas que iniciaron su exploración.

De este modo, las cumbres del Mont Blanc y el Monte Perdido y los nombres de Saussure y Ramond son los grandes hitos de la conquista de la montaña europea $\mathrm{y}$, por consiguiente, de su conocimiento y análisis.

Siguen a esta etapa, sólo refiriéndonos a nuestra zona y hablando a grandes rasgos, los escritos y mapas que Franz Schrader dedica a la región de Gavarnie y Monte Perdido. Schrader ha sido el gran geógrafo del Perdido. Si Ramond localiza el Monte Perdido, descubre los caracteres geológicos de las Sorores y asciende el pico, Schrader le dedica ímprobos esfuerzos a través de los años -desde 1.866 hasta 1924- describiendo minuciosamente sus pliegues, angosturas, collados y glaciares. Con su orógrafo, su barómetro y su visión de agudo dibujante elaboró una documentación precisa a partir de las observaciones.

Aunque ya Lucas Mallada (1878) había apuntado los aspectos más importantes que planteaba geológicamente el Pirineo de Huesca, el trabajo clásico sobre estas montañas, que marca las líneas maestras que seguirán los autores posteriores, es el de Dalloni (1910). Limitándonos al Circo de Tucarroya y a su entorno meso-cenozoico, el estudio clave, que descubre las pautas decisivas sobre las que se asienta este gran edificio montañoso, es el que aporta Peter Misch (1934), referente a la estructura de los Pirineos meridionales medios.

También de los años 30 son las primeras aportaciones españolas al estudio morfológico de la Cordillera. Podemos destacar la que García Sainz (1930), dedica al glaciarismo cuaternario del Pirineo y, de modo especial, la que publica Gómez de Llarena (1936), y que constituye un breve pero interesante estudio sobre el Glaciar Septentrional de Monte Perdido.

Es en 1946 cuando Francisco Hernández Pacheco y Carlos Vidal Box llevan a cabo un estudio centrándose en la observación del 
macizo de Monte Perdido y sus cumbres inmediatas. Atienden a su tectónica, su morfología y muestran gran interés por el "gran testigo geológico" que es el glaciar de Monte Perdido.

En la década de los 50 la revista Pirineos publica monografías y trabajos cada vez más especializados, en los que con frecuencia se tocan temas sobre tectónica, gliptogénesis, etc... de los más altos rincones pirenáicos.

El mismo estilo y dedicación que Pirineos tiene en Francia 1a Revue Géographique des Pyrénées et du Sud-Ouest. Es aquí donde se encuentran aportaciones tan señaladas como las de Boyé (1949, 1950, 1952), Taillefer (1952), Barrere (1952) Brunet (1956), etc. Por los mismos años se realizan investigaciones geológicas sobre la zona calcárea de Gavarnie-Monte Perdido: L.U. de Sitter (1956), con sus cortes geológicos transversales de los macizos pirenáicos Viers (1960), que atienden importantes aspectos del relieve pirenáico; J.G.J. Van der Lith (1965), que estudia el Manto de Gavarnie; Souquet (1967), que analiza la estructura general del Macizo de las Tres Sorores (Monte Perdido); Van de Velde (1967), que se interesa por la geología de Ordesa; Seguret (1969), Choukroune y otros (1968) tratan las características del manto de Gavarnie; Soler y Puigdefábregas (1970), estudian las líneas generales del Alto Aragón Occidental.

La bibliografía geológica es abundante; sin embargo los aspectos geomorfológicos han sido, hasta el momento, insuficientemente tratados, no habiéndose llevado a cabo un verdadero estudio de este tipo en las prominencias más relevantes de la Cordillera; en Tucarroya y el macizo de Monte Perdido-Marboré, contamos sólo con las breves notas de Gómez de Llarena, Hernández Pacheco, Vidal Box, Boyé y Barrère.

\section{Articulación general del relieve}

Estos edificios montañosos presentan un neto carácter estructural, tanto en el gran mu ro escalonado de Monte Perdido y en los tajos del Cilindro como en la posición vertical de los estratos calizoareniscosos del Maestrichense que forman la aguda crestería AstazúPico de Pineta.

A su vez, la cresta se ve afectada por frecuentes fracturas que 
originan cortes y brechas tan importantes como la de Tucarroya. El desnivel desde la base hasta su culminación es mucho menor que en la vertiente opuesta del Circo ( $800 \mathrm{mts}$.), no sobrepasando nunca los $300 \mathrm{~m}$., que, por otra parte, están generalmente expuestos a solana.

El fondo del Circo de Tucarroya es una alta plataforma, que desde el escarpe conocido como Balcón de Pineta, a 2.450 mts., se eleva paulatinamente durante unos $3 \mathrm{~km}$. hacia el NO., para acabar en el collado fronterizo de Astazú, a $1.975 \mathrm{mts}$. Dicha platàforma no es absolutamente llana, sino que se ve toda ella y en especial su porción nororiental, ocupada por resaltes rocosos que forman cuestas monoclinales, (en ocasiones flancos de pequeños pliegues), de relevante importancia morfológica. Estas cuestas, de frentes longitudinales al eje mayor del Circo, modeladas sobre el mismo paquete maestrichense que materializa la cresta de Tucarroya, poseen una mayor importancia en el contrafuerte topográfico que, apareciendo bajo el collado de Astazú, deja el Lago al N. y va a morir sobre el camino de acceso al Balcón de Pineta.

Allí se localiza longitudinalmente una cresta, más o menos continua que, dentro de la gran complejidad tectónica del área y participando de diversas inflexiones, alcanza a dividir el fondo del Circo en corredores paralelos. El de menores dimensiones, quedará situado al N-NE. de este resalte, dando base a la crestería de Tucarroya y algo más elevado que el valle principal.

En el área situada bajo la Brecha, aprovechando la excavación ocasionada por el cruce de las fracturas con este valle principal, se instala el Lago de Tucarroya, de Pineta o de Marboré, pues de todas estas formas se le denomina ${ }^{2}$, reembalsado por un dique construido en 1927 por Hidroeléctrica Ibérica sobre su desagüe natural. Es hacia este reservorio donde van a parar las aguas del valle lateral, ya que debido a una cierta contrapendiente no poseen salida directa hacia los cejos rocosos de Pineta.

La disposición geológica del conjunto de Tucarroya-Monte Perdido puede quedar sencillamente explicado del siguiente modo:

2. En el mapa militar 1:25.000 se le denomina "de Pineta o Marmóres"; en el 1:40.000 de ICONA como de Pineta, de Marboré, o de Tucarroya. En el 1:250.000 francés con el nombre genérico de "Lac Glacé". Creemos como más apropiados los nombres de "Lago de Pineta" o mejor incluso "Ibón de Tucarroya", pues son los menos susceptibles de confusión. 


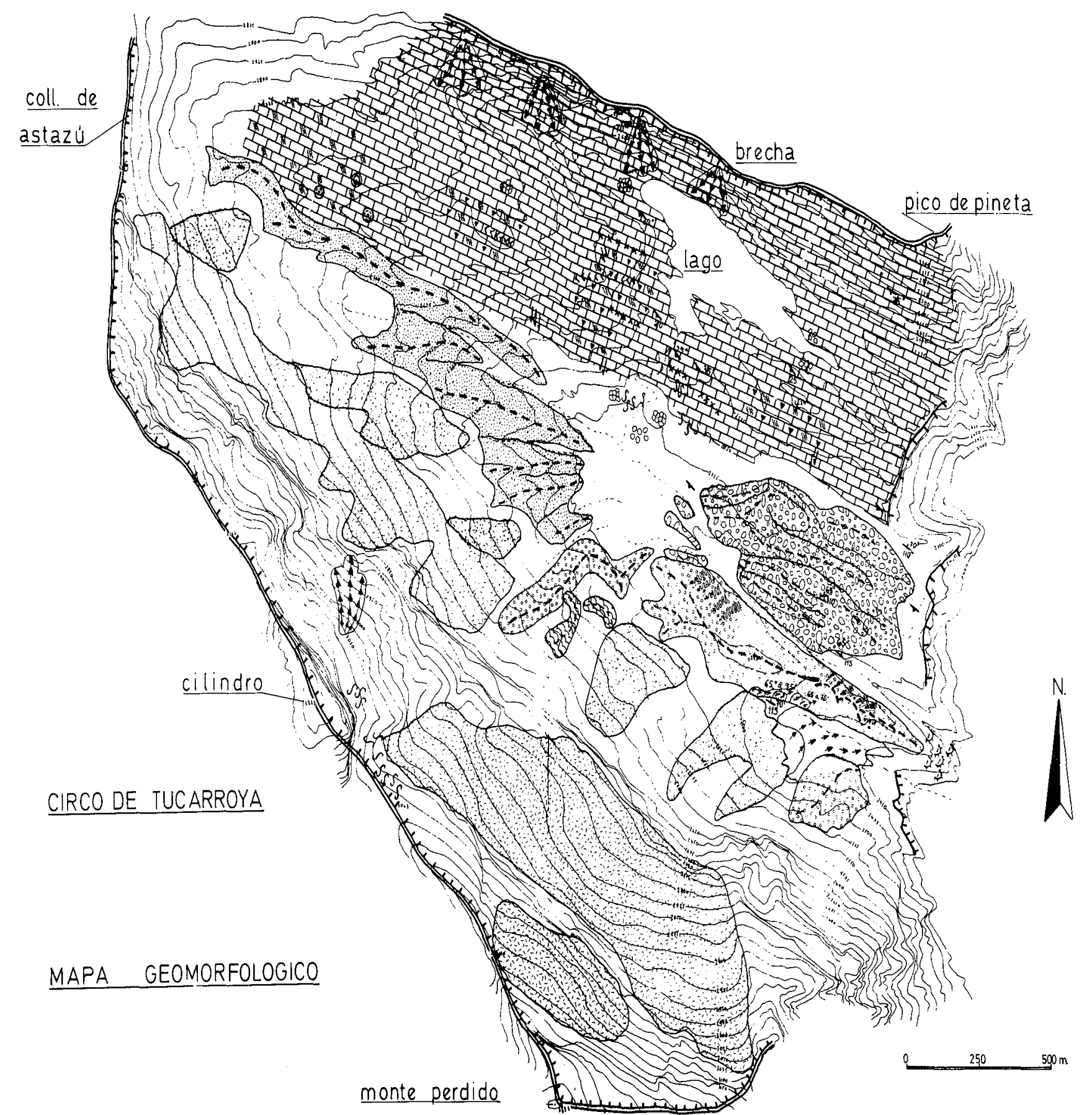

\section{SIGNOS CONVENCIONALES}

FENOMENOS GLACIARES

DENUDACION

ClRCos

$X_{\text {umbrales }}$

ACUMULACION

$\because$ MORRENAS SUBACTUALES DE

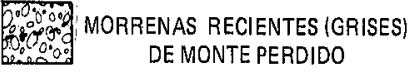

-3 MORRENAS DE CILINDRO

$$
\text { YMARBORE }
$$

FENOMENOS FLUVIOGLACIARES ACUMULACION

$\because \cdots$ MORRENAS DE ABLACION CON CANALES PROGLACIARES

MANTOS PROGLACIARES

\section{FENOMENOS MIXTOS}

MORRENAS RETOCADAS POR LAVADO O SOLIFLUXION

FORMAS SUBORDINADAS

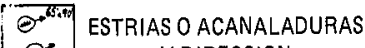

$\odot *$ Y

\section{ROCA DE CAJA}

포몬

CALIZAS MAESTRICHENSES

${ }^{110} / 4$ ESOUISTOSIDAD

is DIRECCION Y BUZAMIENTO $\gamma_{20}$. DIRECCION Y BUZAMIENTO
DE ESTRATOS

$x \times x \times$ EJESINCLINAL

'm?' EJE ANTICLINAL
ACCIONES NIVALES

MORRENA DE NEVERO

ansis? ARROYADA NIVAL

GLACIARES ACTUALES

FENOMENOS KARSTICOS

표민

CAMPO LAPIAZ DESMANTELADO

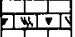

DESMANTELADO

(3) POZO, DOLINA DE HUNDIMIENTO

(Q)

FENOMENOS PERIGLACIARES

$22^{2}$ GELIFLUXION LAMINAR

$/ /$ gelifLusion PElicular

1. DeRRUBIOS ASISTIDOS AMORFOS

CAMPODEPOLIGONOS

$\because$ CIRCULOS DE PIEDRAS

is 
a) Las calizas areniscosas del Maestrichense se asientan sobre las calizas de hiporites replegadas por el empuje de la masa hercínica, y que buzan hacía el S. Todo ello sobre el frente de la masa de empuje. La masa ma estrichense compleja está deformada en anticlinal vergente al S. junto al frente, y un sinclinal al S. cuya complejidad local aumenta según nos elevamos altitudinalmente. En el seno de este sinclinal aparece el Danés-Paleoceno y también el Eoceno.

b) El comportamiento frente a la tectónica diferencia claramente las calizas areniscosas maestrichenses muy adaptables y plásticas frente al resto del conjunto, más rígido y que se resuelve ante las presiones en escamas superpuestas y corridas.

En concreto, todo el gran murallón cumbreño de Marboré-Cilindro Monte Perdido-Soum de Ramond se engloba en lo que podríamos llamar escama del Marboré, conjunto muy replegado de gran complejidad local y que muestra mejor su complicada disposición en las laderas meridionales. En las paredes del Cilindro aparece un anticlinal de eje más alto que los restantes y con el sinclinal alcanzando mayor profundidad. Es en estas alturas donde los pliegues se muestran más vigorosos.

Por otra parte, existen indicios, debido a residuos paleógenos incluidos en el flysch vecino, de la existencia de escamas más altas conformando tal vez un anticlinal inmediato superior al conjunto arquitectónico actual.

Asi pues, la Unidad de Monte Perdido se constituye como una estrecha franja meso-cenozoica enormente perturbada por. la tectónica alpídica en sus distintas fases, con grandes plegamientos y superposiciones de vergencia $S$.

Se han observado 50 líneas principales de fractura, detectables claramente a escala aproximada 1:30000 en fotogeología. De las fracturas observadas, 25 poseen la dirección NE-SSW; 19 siguen la NNW-SSE y 6 muestran dirección ENE-WSW.

\section{Modelado Kárstico}

Como sabemos, la litología presente en Tucarroya y Monte Perdido se reparte entre el maestrichense, las calizas paleocenas del Dano-Montiense y las eocenas del Ilerdiense. Sin embargo conviene 
destacar la existencia de diferentes tipos de calizas margosas, etc. Es indudable que cada una de estas rocas va a tener un comportamiento físico-químico, en detalle, distinto del resto.

Las zonas kársticamente más activas aparentan ser las situadas en los dorsos de los estratos que forman las microcuestas en la prominencia central del fondo del Circo. Estos estratos monoclinales presentan un aspecto muy parecido al de "dientes de sierra", con sus pequeños frentes (de 1 a $15 \mathrm{~m}$.) hacía el $\mathrm{N}$. y con el eje de pliegue en la dirección general ya comentada NW.SE.

Si bien los rincones situados bajo los microfrentes tienen también sus peculiares procesos morfológicos, que serán objeto de comentario posterior, los dorsos de éstos, muestran un campo apropiado para la disolución kárstica. Por un lado el buzamiento en estos estratos es mucho menor que en las cretas circundantes, por otro, la altitud es igualmente inferior. Todo ello hace que las abundantes nieves depositadas sobre ellos, dada su disposición y situación, fundan con relativa rapidez, deparando una humedad mucho mayor que sobre los verticalizados estratos de las crestas, que canalizan las aguas fundentes por brechas y diaclasas.

La estructura es así la que articula la mayoría de las acciones kársticas, ya sea con su diaclasado de detalle o aprovechando las suaves inclinaciones de los dorsos; éstos aparecen además con una superficie meteorizada con numerosas "muescas" relacionadas con su esquistosidad, aprovechadas tanto por la disolución de los carbonatos como por la gelifracción en formas muchas veces combinadas, aunque con predominio de la karstificación: los pequeños lapiaces con profundidad media de $5 \mathrm{~cm}$. aproximadamente, e incluso algunas pequeñas simas, ocupadas en buena parte por la nieve, muestran la inequívoca acción de las aguas sobre las calizas.

El área más afectada por estos hechos es la situada al W. del Lago y al S. del Gran Astazú, en razón de una disposición estructural más suave de los estratos calizos y en una mayor desnudez de estos, al carecer de derrubios que los recubran, aunque también pueda interferir algún factor litológico.

La acción erosiva de carácter químico de las aguas sobre las calizas de nuestra zona, parece no ser realmente hoy muy considerable, ni tampoco haber ostentado el verdadero protagonismo en la formación del relieve del área.

Recogiendo los valores teóricos obtenidos por Nicod hemos 


\section{MORFOLOGIA MACIZO DE MONTE PERDIDO}

estimado que las aguas de nuestra zona pueden contener una media del orden de $306 \mathrm{mg} / 1$. de total de carbonatos. Si, a su vez, utilizamos para nuestra zona el valor teórico de $1000 \mathrm{~mm}$. anuales de lluvia útil, que consideramos apropiado según nuestras informaciones, $y$ damos para el total de superficie de la cuenca $5,4 \mathrm{Km}^{2}$, tenemos una resultante de $109 \mathrm{~mm} /$ milenio $^{3}$.

Esto parece corroborado por el valor relativamente bajo obtenido para la disolución de las calizas, tomando como base el análisis llevado a cabo en Octubre de $1978^{4}$.

De los resultados de este análisis se deduciría que la banda calcárea desaparecida por disolución podría evaluarse en torno a los $20 \mathrm{~mm} / \mathrm{milenio}$, valor sensiblemente más bajo aún que el calculado partiendo de los datos de escorrentía y estimaciones de disolución utilizados anteriormente.

Esta notable diferencia puede deberse a distintas causas: la primera estimación recoge unos valores medios anuales, mientras la segunda se basa en datos de un mes en que, debido al estiaje, los caudales son mínimos. Por otro lado, la muestra ha sido tomada sin dejar transcurrir el lapso de tiempo usual que la debería separar de las últimas precipitaciones, y a ello puede deberse el carácter muy subsaturado que presentan las aguas.

En cualquier caso, convendría realizar análisis más detallados con muestras de agua tomadas sobre el propio terreno, en diferentes lugares y con verdadera información sobre cuál es la cantidad de lluvia útil recibida en este espacio.

3. El cálculo es el siguiente:

LLuvia útil $=1000 \mathrm{~mm}$. anuales $=1 \mathrm{~m} .^{3} / \mathrm{m}^{2}$

Superficie de la cuenca $=5,4 \mathrm{Km}^{2}=5,4 \times 10^{6} \mathrm{~m}^{2}$

LLuvia útil en toda la cuenca $=1 \times 5,4 \times 10^{6} \mathrm{~m}^{3}=5,4 \times 10^{6} \mathrm{~m}^{3}$.

$300 \mathrm{mg} / 1 . \mathrm{CO}_{3} \mathrm{Ca}=300 \times 10^{3} \mathrm{mg} / \mathrm{m}^{3}=300 \mathrm{~g} / \mathrm{m}^{3}{ }^{3}=300 \times 10^{-3}$ Tonel. $/ \mathrm{m}^{3}$

Masa total de $\mathrm{CO}_{3} \mathrm{Ca}$ evacuada anualmente. $=300 \times 10^{-6}={ }_{54}={ }_{10}^{6}=1620$ tonl. anules.

Densidad $=\frac{\text { masa }}{\text { volumen }}=2,75 . \quad 2,75 \times \mathrm{V}=1620 \mathrm{Tm}$.

$\mathrm{V}=1620 / 2,75=589,09 \mathrm{~m}^{3} \mathrm{C} \mathrm{O}_{3}$ Ca por año.

$\frac{589}{5,4} \mathrm{~m}^{3} / \mathrm{Km}^{2} /$ año $=109 \mathrm{~mm} . / \mathrm{milenio}$.

4. Estos análisis se llevaron a cabo el 17 de Octubre de 1978 por el Centro de Análisis de Aguas S.A. sobre una muestra recogida en un arroyo de las paredes de Pineta, el 11 de Octubre de 1978. 
Dentro de este contexto se presenta también el problema del origen del Lago o Ibón de Tucarroya, que podría ser glaciar, estructural o kárstico (o resultado de una combinación de diversos factores). Si abogásemos por un carácter eminentemente kárstico (como algún autor ha apuntado), esta afirmación se habría de ver refrendada por. los correspondientes análisis de sus aguas. No obstante, su posible origen por disolución preglaciar queda como algo meramente especulativo, ya que con los análisis sólo se podría obviamente deducir si en la actualidad la disolución es suficiente para que en un lapso lógico tiempo se hubiera podido formar la cubeta lacustre. En cualquier caso; todas las observaciones confirman la confluencia de diversos factores, como rumbos de fracturas, acción glaciar, etc. en el modelado de la cubeta del Lago.

E1 modelado kárstico está, no obstante, a un nivel u otro, siempre presente en la zona. Su acción se manifiesta en forma de microlapiaces, simas, surgencias, etc., si bien nunca con el desarrollo que presentan estas formas sobre las calizas masivas de la vertiente sur de las Tres Sorores y Marboré.

En Tucarroya, el karst tiene una indudable trabazón estructural, aprovechando diaclasas, fracturas horizontales o la esquistosidad paralela al eje de los pliegues: Por otra parte, presenta mayor incidencia en aquellos lugares especialmente apropiados, como son los dorsos suavemente inclinados, desprovistos de nieves perennes $\mathbf{y}$ directamente sometidos a la escorrentía de fusión, a la acción de las lluvias, etc.

En general, sobre las calizas grises cenozoicas los procesos parecen acentuarse, pero en nuestra zona este hecho se ve dificultado por los continuos escarpes y paredes en los que estas calizas aparecen, así como por su altitud, (que se traduce en numerosas días de helada), factores que frenan los procesos kársticos.

Así pues, el karst sobre el Circo de Tucarroya es un hecho extendido y notable, pero carente, al parecer, de la fuerza modeladora que ostenta sobre otras zonas vecinas.

\section{Procesos Glaciares}

El glaciarismo tiene un lugar preeminente en el terreno estudiado. Es indudable que en la última glaciación cuaternaria toda el área 
estuvo ocupada por los hielos, aunque el espesor de éstos es hoy dificilmente determinable.

En todo caso la huella glaciar es muy notable y aún más si se piensa en el carácter del sustrato rocoso, muy susceptible a los ataques erosivos.

A) Procesos pleistocenos.- Diversos autores sitúan el glaciar del Würm hasta el lugar donde hoy se encuentra el pueblo de Bielsa, [Hernández Pacheco y Vidal Box (1946)], donde quieren ver lo que fue su morrena frontal. También citan restos morrénicos en las orillas del Cinca en Pineta y en las cercanías de Parzán ${ }^{5}$, y una morrena lateral en Espierba.

Otros hablan de un flaciar mucho más desarrollado que llegaría hasta el desfiladero de las Devotas, tras unirse en el del Valle de Pineta - Cinca - con el de Barrosa y ambos al proviniente del actual valle del Cinqueta ${ }^{6}$.

El cerrojo calizo sobre Bielsa, tajado por el Cinca en fuerte incisión muestra un depósito muy empastado de rocas heterométricas. La relación con aportes de ladera, hoy poco visibles por el bosque, parece indudable. Por ello comprendemos las dificultades que los distintos autores han tenido para dilucidar el verdadero origen de este resalte, aunque sí parece muy factible su filiación morrénica.

Los depósitos visibles en la boca norte de la "foz de las Devotas" son atribuibles tanto a materiales de ladera colgados, como a terrazas de un diferente estadio fluvial del antiguo Cinca, incluso a ambas causas. Aunque también pudieran relacionarse con posibles morrenas, parece aventurado, sin embargo, afirmar su completa filiación glaciar.

Así pues, al contrario de lo que ocurre en otros grandes valles meridionales del Pirineo, en el del Cinca el área total ocupada por los hielos cuaternarios en su máxima extensión, o al menos por el aparato würmiense, es difícil de discernir con precisión y ello haría necesario un estudio particular sobre este tema concreto.

En cualquier caso y aunque sólo fuera por su claro perfil en " $U$ ", así como por analogía climática con los valles paralelos vecinos, el valle de Pineta se hace heredero de un potente modelado glaciar sin ningún lugar a dudas. La incógnita queda planteada para la porción

5. Memoria Geológica n..$^{0}$ 14. Viella, del Mapa Geológico $1: 200000$, I.G.M.E. pág. 22.

6. Gómez de Llarena (1936), cita a Penck como mantenedor de esta interpretación. 
del valle del Cinca entre Bielsa y las Devotas, aunque es muy posible que el frente del máximo würmiense no alcanzase la confluencia con el Cinqueta.

En cualquier caso, sirva esta ojeada para tomar idea de las colosales dimensiones de los glaciares que ocuparon la zona, y de cómo el Circo de Tucarroya, su más alto baluarte, debió funcionar como un receptáculo alimentador, recogiendo nieves y acumulando hielos, que posteriormente, y tras unirse a los almacenados en La LarryLa Munia, al NE., fluyeron ya por el valle de Pineta, formando el Glaciar del Cinca.

Así pues, parece evidente que es en el álgido glaciar cuaternario cuando se "esculpieron" las principales formas que aún hoy se nos hacen patentes. Sobre la base de las morfoestructuras terciarias, que dispusieron las líneas maestras del Circo de Tucarroya, esas potentes masas de hielo actuaron con enorme vigor.

Podemos distinguir dos zonas de diferentes características de las mofogénesis glaciar en el área de Pineta, separadas por el gran escarpe de las "paredes", de unos $800 \mathrm{~m}$. de desnivel; la zona inferior (valle de Pineta) y la superior (Circo de Tucarroya).

a) Parte alta del valle de Pineta: El gran escarpe de las paredes de Pineta, debió verse cubierto por masas glaciares fracturadas y caóticas, dando uno de los glaciares pirenáicos más tortuosos y complejos. Este a su vez debía caer en pendiente cascada sobre el de la Munia y esto parece ser así en función de la actual disposición de los valles.

La base estructural del Balcón y de las paredes de Pineta parece bastante clara. Fuertes y marcadas fallas sobre las paredes del pico Felqueral y Monte Perdido son elocuentes a este respecto. La violenta tectónica a que ha sido sometido el roquedo y el tipo de procesos erosivos de dominante mecánico, como la acción glaciar, que han actuado sobre él, han favorecido la existencia de escarpes acusados.

Esta topografía no es exclusiva del Circo de Tucarroya, y así, el pequeño Circo de Serradets, en el vecino Circo de Gavarnie, presenta una gran analogía con ella, tanto en su situación como en orientación y disposiciones.

El hecho de que el escarpe del Balcón no muestre una clara morfología glaciar de detalle está en razón de que la fuerte pendiente 


\section{MORFOLOGIA MACIZO DE MONTE PERDIDO}

debió permitir tan sólo una lengua en cascada, incluso con escalones rocosos, frecuentes desplomes de hielo y una acusada inestabilidad a la que contribuiría su exposición S.E. Por todo ello la elaboración de huellas glaciares de abrasión -no así de desalojo-debió ser mínima o desaparecieron rápidamente, ya que al iniciarse los retrocesos glaciares las frecuentes paredes que jalonan la pendiente fueron retocadas por la meteorización, periglaciarismo, desplomes y torrentes. Esta pendiente presenta hoy diversas formas, algunas incluso, como ciertas exhumaciones kársticas, probablemente preglaciares: canales de avalancha, "gleras", niveles de cavernas en las calizas kárstificadas, paredes, conos de deyección, etc., que reflejan un abanico de procesos morfogenéticos relacionables con la gran pendiente. Como consecuencia de ello, los depósitos situados en la base de las paredes de Pineta, de gran heterogeneidad, están constituidos por caos de bloques de diverso origen (gravedad, morrénicos, torrenciales), y de distintas etapas (finiwürmiense y holoceno).

Partiendo de la evidente instalación de los hielos sobre el gran escarpe, estos debieron excavar sensiblemente su zona inmediata inferior, como corresponde a un lugar con tan acusada potencialidad erosiva, tanto estructural como glaciológica. En la retirada de los hielos, es previsible, pero no constatable, que se produjera una importante deposición, (tal vez en algún momento de carácter lagunar), que iría colmatando el cuenco inferior de las Paredes.

Este cuenco, sometido a la acción torrencial posterior, mostrará, en los cortes del depósito, un importante espesor de sedimentos varios y empastados donde abundan los bloques.

Aguas abajo, donde hoy se sitúa el puente sobre el Cinca, se encontraría el umbral calcáreo de cierre del cuenco. Aquí el río se encaja profundamente en la roca caliza y los "diques" del cerrojo están actualmente enmascarados por la vegetación, que ya es boscosa. Esta hipótesis puede verse apoyada por el perfil del curso del Cinca desde el Balcón hasta el valle de Pineta. Un estudio detallado del área de sedimentación que recogía los aportes de las paredes puede constituir una interesante fuente de información sobre la evolución morfológica reciente del sector.

Como prueba de la potencialidad morfogenética que presenta el abrupto de las paredes de Pineta basta mencionar la avalancha de nieve que se produjo en la primavera de 1978, tras una semana de fuertes nevadas. Iniciada sobre los $2.200 \mathrm{~m}$., alcanzó la cota de 
1500 m., con más de cincuenta metros de anchura, y llegó a taponar toda la incisión del cauce del Cinca, que alcanza en algunos lugares más de $30 \mathrm{~m}$. de profundidad; los restos de este alud permanecieron durante todo el verano en una cota muy inferior a la de las nieves permanentes.

b) Circo de Tucarroya: Este Circo muestra en líneas generales una morfología típica de carácter glaciar, si bien muy influenciada por las estructuras, que en algún caso complican y atipifican el modelado. Es indispensable, no obstante, preguntarse aquí también por la importancia de la acción periglaciar, anterior a la instalación de los hielos, y, en relación con las teorías de Boye (1949), sobre la necesidad de tal acción para que los glaciares puedan excavar.

El gran glaciar que, desde el collado de Astazú, vertía sus hielos hacia Pineta debía recibir sus aportes principales de la vertiente septentrional de Marboré-Cilindro-Monte Perdido, pero también el recuenco bajo el gran Astazú y Tucarroya-Pineta presentaba un importante casquete helado, que rebosaba sobre la escarpada pared sintuada encima del "embudo", por donde transcurre el actual sendero de acceso.

Probablemente, en el máximo glaciar ( ¿Riss?), se produce ya el excavado general y el de la cubeta del Lago, y sobre todo, la conformación de la actual morfología del Circo: su excavación central, la disposición de sus escalones laterales, etc.

Pero, aunque creemos probable que pudiera ser en el Riss cuando se labran las mofoestructuras glaciares, sin duda nos consta que el Würm las utiliza y que es precisamente en este momento cuaternario glaciar y finiglaciar, cuando quedan definidas las líneas generales del relieve que, con leves alteraciones, llega a nuestros días.

Pero si estas modificaciones morfológicas últimas son menores, dentro de las grandes pautas o estructuras, no lo son en cuanto a su papel en la configuración definitiva del paisaje de Tucarroya. E1 último retoque morfológico del paisaje comienza realmente incluso con la retirada de los hielos.

Pero todo el proceso morfológico glaciar, y con él el verdadero origen del valle, está aún supeditado a la discusión planteada entre las tesis aprobatorias de la excavación glaciar por sí misma, y aquellas otras que la niegan o la condicionan a circunstancias muy determinadas. 
Como es sabido, existe una tendencia encabezada cronológicamente por Boye $(1949,1950)$, Lliboutry y Vivian (1976), que fue difundida extensamente por Tricart, y Cailleux, según la cual sólo el aborregamiento, el pulido y la estriación son de origen glaciar, pero no así la excavación, y, en los casos en que ésta aparece, sería debida a una fragmentación periglaciar previa del lecho que después ha podido sufrir un desalojo por los frentes glaciares, actuando en forma de bulldozer. En algunos casos, Boyé acepta la suma de efectos tectónicos y kársticos para el modelado de las cubetas y propone el Circo de Tucarroya como uno de los ejemplos donde su hipótesis se cumple.

Frente a esta idea, otros autores, de Matthes a Taillefer (1952), creen en la posibilidad de que los glaciares elaboren su relieve a partir de ciertas estructuras previas que les condicionan, operando por tanto, tales estructuras como factor principal y determinante para la elaboración de un relieve de excavación glaciar.

Según Martínez de Pisón y Arenillas (1976) las distintas teorías sobre el glaciarismo están en relación directa con la importancia que se quiera otorgar a las estructuras para la explicación de la morfología glaciar. A pesar del indudable protagonismo que las pautas estructurales ejerce, estos autores señalan: "no pretendemos abogar por una solución eminentemente tectonista del proceso glaciar. Es evidente que otros factores (litología, climatología, dinámica del glaciar, etc...) actuando conjuntamente con la tectónica, intervienen en la resolución del problema global". El fenómeno de la sobreexcavación glaciar está, pues, dentro de toda lógica comprobable, si bien se ejerce fundamentalmente sobre zonas más o menos tectonizadas.

Sin embargo, como ya indicamos, el mismo Boye (1952) intentó una interpretación del modelado del Circo de Tucarroya basándose en sus propias hipótesis, destacando sobremanera la acción periglaciar, gelivación en este caso, como agente erosivo fundamental. De este modo, el relieve en pequeñas cuestas, que él considera periglaciares y preglaciares, habría sido sólo "retocado" por los glaciares. La sobreexcavación del Circo, y en concreto del Lago, se podría deber, en todo caso, a la unión de fectores tectónicos y kársticos, unidos a la gelifracción, pero Boyé nunca apunta la posibilidad de una acción directa de los hielos glaciares.

En concreto, en el circo se pueden distinguir dos espacios dis- 
tintos, en relación con los procesos glaciares: Uno, lo constituirían los flancos septentrionales del Cilindro-Monte Perdido, con los cambios litológicos que se producen en ellos, su pendiente, su orientación y su altitud. El segundo ámbito de acción del modelado glaciar sería el verdadero fondo del Cinco o "valle colgado" de Tucarroya, con una homogénea y característica disposición estructural.

En el área más alta, el modelado podríamos calificarlo de más adaptado a la estructura, ya que se conforma en escalones relaciónados con la misma. Los escalones se establecen en zonas de contacto del Maestrichense con el Danés-Eoceno, que ofrecen áreas de debilidad (¿tal vez aprovechadas en algún caso, donde fuera posible, por el periglaciarismo preglaciar?) para la acción de los glaciares. La observación al N.-NW. del Glaciar inferior de Monte Perdido, en contactos entre distintas litologías deja ver como éstos aparecen sometidos a un intenso modelado, ya sea periglaciar, glaciar, o tal vez más bien, de ambos.

De todos modos, los glaciares de esta ladera, y fundamentalmente el Perdido, se emplazan en un relieve común al de los escalones o gradas del Circo de Gavarnie, al de las vertientes meridionales de las Tres Sorores, etc., que hoy presentan múltiples acciones erosivas que van desde el sobreexcavado, localizado en las áreas citadas, a fuertes procesos de gelivación sobre escarpes humedecidos por aguas de fusión. Estos procesos están, además, reforzados por la gravedad.

Pero todos estos relieves obedecen prioritariamente, como es lógico, a la poderosa trama estructural del conjunto, y los procesos morfogenéticos típicos de la alta montaña han seguido la trama, destacando incluso sus elementos de un modo peculiar, de manera determinante. Las alineaciones de los estratos, los ejes de los pliegues de mayor o me nor rango, las esquistosidades, las diversidades litológicas, la fracturación, la disposición morfoestructural del edificio en su conjunto, los efectos, en suma, de la tectónica del manto de Gavarnie sobre el gran paquete rocoso de las Sorores es lo que ha dirigido el modelado en general y en cada detalle.

E1 relieve preglaciar - del que no quedan materialmente huellas- debió ya seguir esta pauta y por ella se canalizó la acción glaciar. Esto no es constatable, pero sí razonable. Y no es constatable porque en todo el ámbito estudiado sólo existen huellas gla- 
ciares y posteriores; todo el conjunto Tucarroya-Sorores ha sido modelado por los hielos. La última glaciación ocupó completamente la superficie del circo, por lo que todas las formas glaciares heredadas proceden de ella; si el circo fue ya parcialmente modelado con anterioridad por períodos glaciares previos al Würm, puede ser suponible, pero no es demostrable. Una preparación periglaciar prewürmiense del roquedo es también razonable, pero sin conceder a tal preparación ni un papel clave, también indemostrable. en un dudoso "defonçage proglaciar", ni una eficacia modeladora capaz de elaborar por sí sola un circo de tales dimensiones, ni una supervivencia de sus formas preglaciares en el fondo del recuenco a través de todo el período glaciar y el dinámi co holoceno de Tucarroya.

Los efectos del periglaciarismo en el roquedo de Tucarroya son abundantes, pero "peculiares", lo que no facilitaría una excavación ni, aún menos, una sobreexcavación. Suponer, por otro lado, un "defonçage" múltiple de un aparato de limitado tamaño y muy pulsador acantonado en el Circo no parece responder a la reconstrucción del glaciarismo pleistoceno, responsable de las grandes formas altopirenáicas, a que conducen todos los hechos morfológicos.

En cambio, la disposición estructural del roquedo no sólo guía los valles y el karst preglaciares y, después, la acción de los aparatos en ellos, sino que permite, en conexión con fracturación, diaclasamiento, esquistosidad y litología, un desalojo de materiales por el glaciar, del que resultan las formas hoy existentes.

La caja del circo es netamente estructural, entre la cresta tectónica de Astazú-Pineta y la mole de pliegues apilados de las Sorores. El conjunto intermedio -Tucarroya- presenta condiciones propicias para una alta cuenca de recepción con salida natural a Pineta, siguiendo el rumbo de los estratos y la inclinación general del edificio. En el contacto con Pineta encuentra una línea de fractura importante casi ortogonal, que es parcialmente aprovechada por el torrente de La Larri y que permite una importante excavación local, donde reproduce el codo que tal torrente realiza al adaptarse a los rumbos del roquedo y dar lugar al gran valle subsecuente de Pịneta. Este hecho va a ocasionar a su vez un efecto de umbral en época glaciar, que acentuará el desnivel existente y su pendiente hasta construir el escarpe entre Tucarroya y Pineta. Los cruces de fracturas con las alineaciones sedimentarias en Tucarroya van a ser 
un lugar propicio para la gelifracción de los "nunataks" y el desalojo definitivo con tajos, agujas, etc., que se entrecruzan con las formas resultantes de las alineaciones sedimentarias (cuestas, dorsos de flancos, peldaños); aparte de la incidencia de la erosión diferencial de los cambios de nivel de eje, el cruce de importantes fracturas con dichas alineaciones va a permitir un sobreexcavado, por especial intensidad local del desalojo subglaciar en área especialmente apta al desgajami ento de bloques, que acabará por labrar el recuenco donde hoy se aloja la laguna o ibón.

No es, por tanto, casual que aparezca relacionado tal recuenco con otras formas asociadas, como la brecha de Tucarroya, que aprovecha una misma fractura, y a una especial densidad de fracturación. Posteriormente, los retoques holocenos han modificado el detalle con morrenas tardías, glaciares póstumos, y reactivados recientemente, depósitos de ladera, torrencialidad, procesos crionivales y kársticos. Este retoque, no obstante, no ha sido intenso y continúa ejerciéndose según la trama estructural.

Por otra parte, cabe preguntarse, dada la existencia de glaciarismo en la actualidad en Tucarroya, si este alto recuenco estuvo o no libre de hielos en los interglaciares y en qué medida $\mathrm{y}$, concretamente, en el Riss-würm, que según algunos autores fue más breve de lo que se venía señalando. La morfogénesis glaciar parece, así, un hecho decididamente dominante.

En todos los casos el flujo de estos hielos fue más o menos paralelo al eje general de los estratos del fondo del Circo y su escorrentía, fluyendo por toda la superficie, ejerció su abrasión sobre las culminaciones de los frentes de cuesta, mientras, diferencialmente pudo abrir pasillos subsecuentes. Todo lo cual contribuyó a resaltar el actual relieve en cuestas, pues los masivos dorsos de estos estratos se vieron mucho menos afectados por la acción del hielo.

Pulimentos y estriados de esa primera época pueden haber desaparecido, por su superficialidad, sobre materiales tan suceptibles a la erosión, tanto química como mecánica.

B) Procesos glaciares recientes y actuales.- Las huellas de los últimos fenómenos glaciares, si bien no son de la envergadura de los tectónicos ni de los paleoglaciares, se aprecian claramente, e incluso otorgan al paisaje su más llamativa característica. 


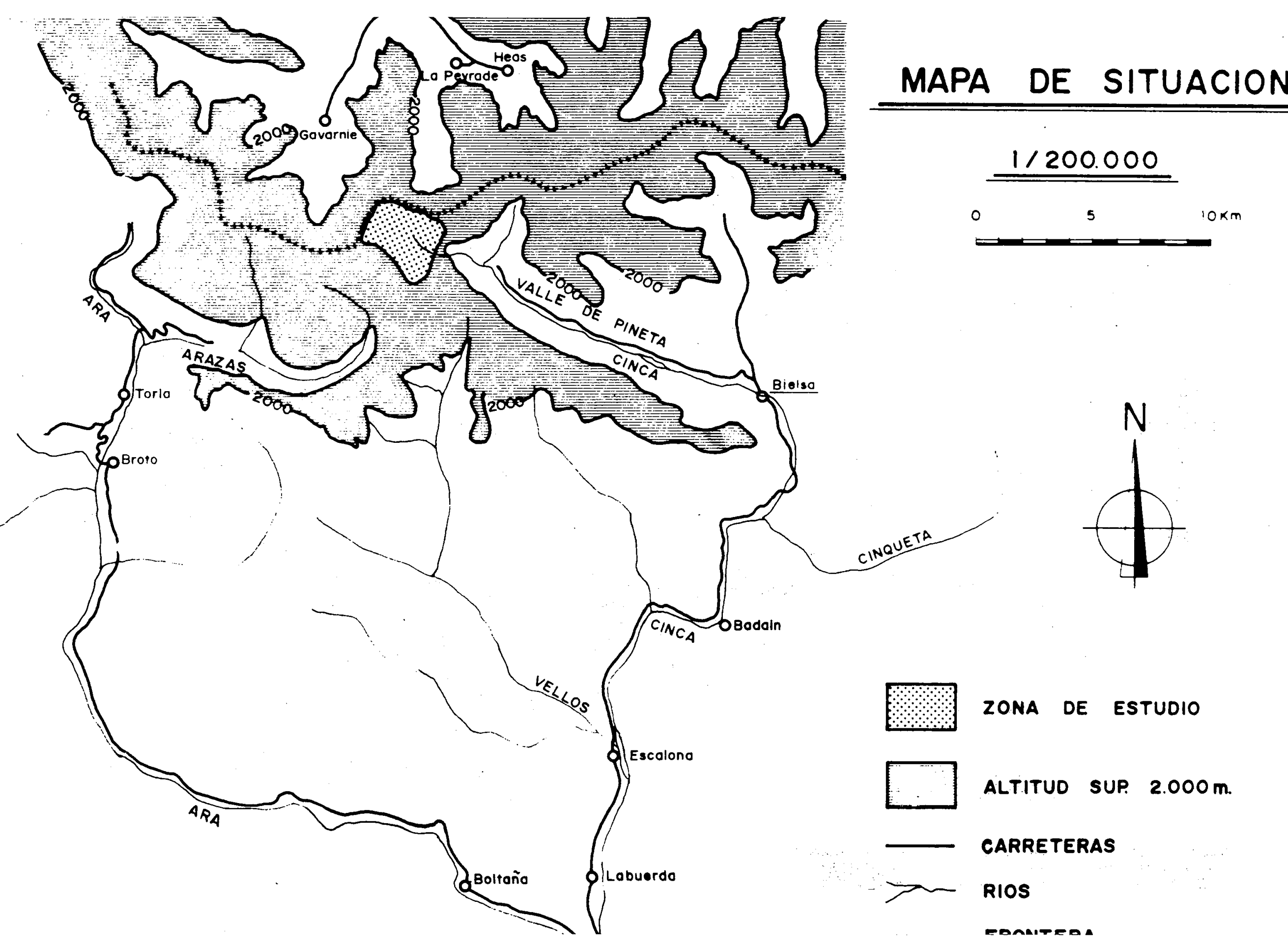




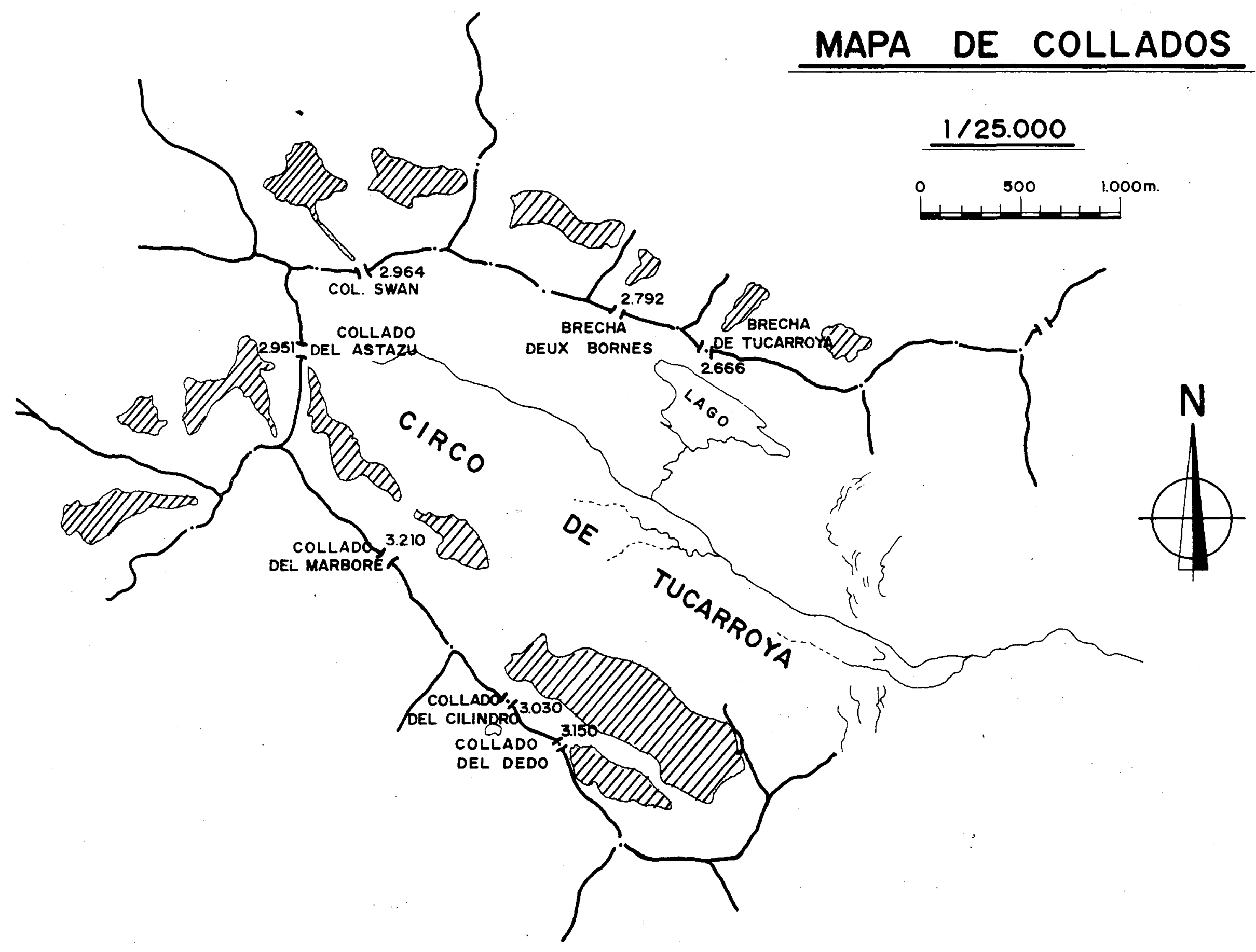


Hay que situar dentro del holoceno a todas las morrenas que aparecen en el Circo, siendo ya históricas las emplazadas en la proximidad de las actuales masas de hielo.

a) Morrenas grises de bloques: Próximas al Balcón y a la Cruz de Pineta, las morrenas son anteriores al resto de las que se encuentran en el Circo, como indican su composición, litológica, posición, tamaño y estado de conservación.

$\mathrm{Su}$ localización es resultado de una deposición frontal (y quizá fronto-lateral) de la masa glaciar del Perdido, con la que confluirán hielos procedentes de las rampas inferiores del Cilindro, situándose sobre los flancos de los estratos que dan la elevación interior del Circo. Su carácter frontal es deducible no sólo de su emplazamiento, sino también en parte de la incurvación hacia el collado del Cilindro, que toma su porción más occidental, sobrepasando sus característicos bloques el curso del Cinca hacia el sur, si bien ya de forma aislada.

Este conjunto morrénico, de bastante anchura y potencia, se apoya sobre los dorsos y culminaciones de las minucuestas maestrichenses $y$, de este modo, existe una apariencia de diversos "vallums" morrénicos yuxtapuestos, que no se pueden interpretar como estadios, ya que realmente sólo indican la presencia de un conjunto de barras estructurales, sobre las que se apoyan los depósitos. Según el número de cuestas que aparezcan localmente -pues debido a la complejidad tectónica de detalle las barras surgen o se extinguen-, la morrena muestra de 2 a 5 crestones de apariencia muy similar a los "vallums" morrénicos, aunque las cuestas dejan ver en frecuentes afloramientos la roca "in situ".

Los bloques que forman los materiales son calizas grises, con gran homogeneidad litológica, relacionables con el Danés-Paleoceno o con el Eoceno de las paredes altas de Monte Perdido y el Cilindro. El tamaño de los bloques, en relación con los restantes del Circo, es grande, siendo frecuente que alcancen de 6 a $8 \mathrm{~m}$. de altura, e incluso más. Los procesos kársticos así como la gelifracción, han afectado de modo notable a todos estos sedimentos.

El origen de esta morrena puede estar tanto en una excavación del lecho como en un escarpe rocoso puesto al descubierto $\mathrm{y}$ atacado por la gelifracción, cuyos bloques desgajados han sido transportados por el vehículo glaciar medio inferior. En el resto del Circo no se aprecian morrenas coétaneas a ésta, al menos de 
apariencias similares. La causa debe buscarse en las características litotectónicas de los escarpes situados al sur, esto es, en la muralla Marboré-Cilindro-Monte Perdido. Mientras el Marboré carece de otra litografía que no sea la maestrichense, el cilindro posee calizas grises terciarias, pero en paredes verticales enmarcadas por fallas que tal vez nunca se vieron tapadas por los hielos.

Por el contrario, Monte Perdido se dispone en los escalones que ya conocemos, con importantes abruptos entre sus plataformas. Dado que su escarpe calizo inferior muestra otro tipo de roca, los bloques de las morrenas en cuestión tienen un origen más alto, en el escarpe que se encuentra bajo el glaciar superior o en los zócalos calizos que se sitúan bajo la antecima.

Hernández Pacheco y Vidal Box (1946) asignan un origen erosivo glaciar a esos bloques de la morrena; nosotros dudamos que esos bloques sean fruto del excavado, y nos parece más probable que constituyan gelifractos caidos en el glaciar, ya que bajo el antiguo glaciar medio, se sitúa el cambio de litología, lo cual habría ocasionado que los cantos de la morrena fuesen de los dos tipos de roca dominante y no de uno solo.

Por otra parte, la desaparición del extremo occidental de la morrena puede deberse a la acción de las aguas corrientes en un momento de mayor potencia erosiva o debido a un estadio de fuerte fusión en los glaciares superiores del fondo del Circo.

Esta morrena parece análoga a la señalada como tardiglaciar por diversos autores en otros Circos pirenáicos. Puede también relacionarse con la también fase fría post-würmiense -seguramente el mismo tardiglaciar- que ocasiona amplios tapizados de derrubios periglaciares en numerosas vertientes del alto pirineo aragonés, concretamente en la vecina cubeta de La Larri, donde tales derrubios recubren el cuenco de excavación glaciar y están recortados por el torrente actual.

b) Otras morrenas del Circo de Tucarroya: El resto de las morrenas que aparecen sobre el Circo muestra un aspecto muy parecido entre ellas, lo cual no permite afirmar, en principio, que sean de igual edad.

El mapa de Schrader de 1874, sitúa el glaciar de Monte Perdido y del Cilindro-Marboré en posición intermedia entre la morrena gris, ya citada, y la más próxima al glaciar actual, que es sin duda más reciente. Los hielos, en dicho mapa, parecen, pues, situarse, a 
punto de retroceder del actual cauce del río Cinca en la parte inferior del Circo, mientras valle arriba llegan casi a remontar el crestón central. Es necesario recordar que Schrader encontró las formas muy cubiertas por la nieve en su visita al macizo del Perdido, lo que pudo confundir y disimular las verdaderas y reales dimensiones de los glaciares.

Por otro lado, parece cierto que, a pesar de su idéntico aspecto, la morrena fronto-lateral izquierda del Perdido, bien visible y definida, debe haberse visto alejada de los hielos con posterioridad a buena parte de las del Cilindro y Marboré, aunque dentro de una misma etapa de retroceso glaciar.

Así pues, más próximas a las Tres Sorores encontramos otra familia de morrenas, más numerosas, de aparatos más pequeños y escindidos, de gran heterogeneidad en el tamaño de los cantos y también en su litología, si bien coinciden todas en estar culminadas por calizas rojizo-ama rillentas ma estrichenses con tamaños regulares, aunque este hecho se observe especialmente en la del Perdido.

En el mapa morfológico hemos señalado la disposición de estas morrenas; en él se puede apreciar una serie marcando la posición del glaciar inferior reciente del Perdido, con sus morrenas laterales y frontales, dispuestas con gran claridad, en especial estas últimas. Asimismo aparece una "morrena de ablación" en el área de retirada de la masa de hielo inferior.

Bajo el Cilindro y Marboré se localiza un conjunto de morrenas algo menos sencillo, algunas son laterales con cierre en "V", y otras tan solo laterales y aisladas. En total se puede hablar en este grupo de siete edificios morrénicos bien definidos.

C) Morrenas recientes de Monte Perdido.- En los depósitos glaciares recientes de Monte Perdido se pueden distinguir cuatro morrenas bien diferenciadas. Una, a la derecha (E.) del glaciar inferior antiguo, situada bajo las rocas del gran contrafuerte triangular que se eleva hacia el Collado Oriental. Se emplaza en un terreno muy escarpado y pendiente, y a duras penas se mantiene definida, al encontrarse situada en un talud, todo él revestido de material morrénico disperso y mezclado con algún aporte de caidas.

Existe otro gran talud morrénico, en lo que debió ser el frente o punto de mayor escorrentía, por donde hoy fluye el arroyo de fusión. No presenta aspecto de muro, y está afectada por ciertas 
incisiones debidas a los pequeños torrentes que parten de los neveros superiores.

El mejor y más característico edificio morrénico de todo el Circo podría ser la morrena que cierra por el N-NE. el ámbito glaciar de Monte Perdido. Se apoya, como la práctica totalidad de las morrenas del área, sobre los dorsos calizos; sus dimensiones son notables, así como el tamaño de los bloques maestrichenses que la culminan, hecho que puede deberse al descubrimiento de ese cejo calizo inferior, -litología maestrichense-, ya a finales del pasado siglo y principios del actual.

$\mathrm{Su}$ forma es la de un muro morrénico casi perfecto, a no ser por un fuerte "arañado" al que se han visto sometidos sus materiales y que consiste en incisiones, a modo de cárcavas, que desde la cresta descienden por su vertiente exterior, según la línea de mayor pendiente, hacia el cercano Cinca. Este acarcavamiento se debe a la acción de las lluvias y a la fusión de la nieve invernal que abren canales y regatos en su ladera externa. Estos procesos pueden tener una continuidad ya secular.

Por último aparece la morrena lateral izquierda, al W. del antiguo aparato inferior. Parece ser una morrena que participa tanto del aparato del Perdido como del Cilindro y, sobre todo, de los aportes que la zona altamente fracturada (Collado del Cilindro), situada sobre ella, le proporcionan, por lo cual es la única que posee una disposición y orientación distinta al resto.

Entre esta morrena, transversal al Circo, y la frontal clásica no hay una continuidad bien establecida, estando el intervalo ocupado por una zona de pequeñas morrenas aisladas con disposiciones aparentemente caóticas.

El reconocimiento de la zona indica una posible destrucción por gravedad de lo que fueron las morrenas coétaneas a las que estamos analizando. A su vez, el condicionamiento estructural -con pequeños resaltes y recuencos- pudo inducir, durante la retirada de los glaciares, a la instalación compartimentada de pequeños lentejones helados, que funcionaron dando morrenas de "nevé", formadas por el deslizamiento gravitacional de los bloques por la superficie del nevero y su deposición en collar o muro en su zona baja.

D) Morrenas del Cilindro-Marboré.- Estas morrenas son más 
dificilmente observables, dado el recubrimiento de nieve a la que se ve sometida su zona alta, pero se pueden distinguir varios tipos: En primer lugar, morrenas a favor de la pendiente, pero no tan perpendiculares a ésta como la situada bajo el collado del Cilindro, ya que se incurvan aguas abajo en la dirección del flujo glaciar. Algunas de estas morrenas dejan ver perfectamente cúal fue la posición estable del glaciar en retroceso, con un aspecto muy llamativo de "pequeña laguna" y su correspondiente arco de cierre. Presentan materiales de tonos grises en la parte baja de sus flancos y se ven coronadas por las calizas rojizo-amarillentas, lo cual parece indicar una instalación inicial de los procesos que posteriormente pasan a situarse de modo dominante sobre el zócalo maestrichense de la base.

Otras morrenas se apoyan sobre los dorsos de los estratos del conjunto sinclinal. Situadas algo más hacia el N. presentan caracteres muy parecidos a los del Perdido, mostrando a trechos la roca "in situ", si bien con menos rastros de estriación y pulimento.

En resumen, toda el área situada entre el collado de Astazú y el Balcón de Pineta y las laderas septentrionales de Monte PerdidoCilindro-Marboré, se encuentra cubierto de residuos morrénicos muy frescos. Todos los datos llevan a afirmar que estos depósitos pertenecen a una etapa de progresión glaciar distante en el tiempo de la que dio lugar a la morrena de bloques grises karstificados y gelivados, y muy próxima a nuestros días, indudablemente coetánea de la etapa denomi nada "Pequeña Edad del Hielo".

Todos estos hechos son también expresivos de la importancia remodeladora del circo de la glaciación reciente. En cuanto a la acción morfogenética de los glaciares actuales, parece mínima y tan sólo capaz de un cierto pulimento y estriado sobre el subtrato; la observación del frente de hielo no ha mostrado bloques que pudieran indicar testimonios de desalojo, aunque podrían encontrarse dentro o bajo la masa glaciar.

En cualquier caso, la gelifracción sigue aportando cantos y gravas al glaciar inferior, que éste después deja caer al nevero de la base. Aquí podría señalarse un modo de deposición glaciar muy peculiar en el que abundan los bloques del cejo calizo inferior, fracturados en formas de paralepípedo y caidos sobre la uniforme pendiente de la base. 


\section{Procesos periglaciares.}

Un fenóme no singularmente importante en el modelado final del Circo ha sido y es el periglaciarismo. La acción del flujo del hielo, produciendo una característica morfológica glaciar, se ha visto complementada y a veces sustituida por la acción del hielo y deshielo de carácter periglaciar.

La gelifracción consiste, con mucho, en el modo principal de manifestarse el periglaciarismo; ha tenido y tiene en la actualidad una señalada importancia en el Circo de Tucarroya, y tan sólo se ven protegidas de ella las zonas ocupadas por la cobertura nival o lacustre y parte de las zonas ocultas por los glaciares.

Sin duda, esta importancia se acentúa cuando la crioclastia puede actuar sobre zonas tectónicamente predispuestas: fracturas, interestratos, esquistosidad, etc. Asimismo actúa también con especial intensidad en lugares frecuentemente humedecidos, ya sea por los canales de fusión de los neveros, o por la retención hídrica, debida a causas muy diversas.

Buen ejemplo de la eficacia de la gelifracción son los derrubios de origen crioclástico bajo algunas brechas de la cresta de Tucarroya, e igualmente los que se mezclan a las morrenas en la base de las paredes meridionales del Circo, o la importante pedrera o glera, muy desmenuzada, que se sitúa bajo el collado del Cilindro, bordeando los hielos del glaciar inferior de Monte Perdido. Estas pedreras, de cantos finos e inestables, al igual que la situada en la misma cumbre del Perdido, son lógico producto de la combinación entre fuertes alternancias térmicas, frecuentes aportes de nieve y rehielos prácticamente cotidianos.

Las paredes, canales y resaltes bajo el cilindro y el Marboré, son terrenos muy apropiados para la labor disgregadora del hielo, ya que, durante buena parte del año, alternan en estas zonas la nieve y la roca y, con ello, abundan los procesos de fusión y rehielo, lo cual facilita una potente gelifracción.

De todos modos, esta crioclastia se presenta en todo el ámbito del estudio; Boyé (1952) señaló, por ejemplo, su eficacia sobre los frentes de microcuestas próximas al Lago de Tucarroya. En una visita al Circo de Tucarroya este autor observó ya, en una limitada porción del mismo, la importancia del periglaciarismo con efectivo 
creador de formas a pequeña escala; su estudio se centró en la acción combinada kárstica y periglaciar, y señaló cómo la acción del hielo se ve facilitada, y dirigida por la esquistosidad y los planos estructurales, haciendo notar también cómo los pequeños clastos desprendidos de los dorsos se ven transportados tanto por la gravedad como por la solifluxión. Boyé comentó igualmente, cómo estos mecanismos de gelivación depositan los bloques al pie de los pequeños taludes e indicó la formación de franjas y festones de clastos en las pendientes situadas bajo dichos taludes.

Las ordenaciones de clastos que se encuentran en algunos puntos del fondo del Circo son diversas. Pueden encontrarse hileras de piedras de tamaños medios (hasta $10 \mathrm{~cm}$. de eje y con formas apaisadas) sobre el dorso de los estratos, a veces transversales y otras longitudinales al buzamiento.

En zonas húme das, a veces en amplios interestratos planiformes y con materiales finos que retienen la humedad, se encuentran pequeños cantos formando figuras geométricas variadas, de aspecto penta o hexagonal, y que en ocasiones asocian al perfil de su dibujo una mínima orla de vegetación. Cerca del Balcón, incluso en sus primeras pendientes, se pueden hallar festones escalonados con orlas de césped sobre los bordes de las terracitas. Pero, mientras este tipo de acción periglaciar es más común, las ordenaciones de clastos con formas poligonales, a alta escala, son mucho menos frecuentes.

Ya J. Gómez de Llarena (1936) anotó tales fenómenos, en concreto las ordenaciones geométricas periglaciares, en su visita a la zona en Junio de 1935, aportando una interesante fotografía y un hipotético esquema de su perfil. En dicha foto se aprecia muy bien cómo los clastos, siempre muy delgados y de un tamaño ligeramente superior al resto de los que les rodean, se verticalizan, siguiendo una figura cerrada poligonal. Gómez de Llarena no da una explicación de su génesis y sólo hace referencia a las condiciones climáticas a las que se ven sometidas estas formas, así como también a su empastamiento en un barro de descalcificación.

Boyé (1952) comenta, en cambio, la posibilidad de que se asocien a zonas some tidas a retracción, debido a un desecamiento, y cita cómo en Groenlandia, en el 90 \% de los casos en que encontró polígonos, éstos se situaban bajo las aguas de pequeñas lagunillas de fusión. Pero, en otros casos, descarta esta posibilidad; un corte en el terreno muestra cómo las piedras que dan la forma poligonal 
no sobrepasan los $12 \mathrm{~cm}$. de profundidad $\mathrm{y}$, por debajo, el limo está exento de clastos. A partir de un rústico sondeo, Boyé pensó incluso en la posibilidad de existencia de un suelo helado a unos $70 \mathrm{u} 80 \mathrm{~cm}$. y en los alrededores del Lago.

En cualquier caso, teniendo en cuenta la enorme cantidad de días que el suelo se ve recuebierto de nieve, con su conocido efecto de aislamiento térmico, y que, cuando ésta funde, las temperaturas son ya algo más benignas, parece prudente optar por una actitud "desconfiada" ante esta afirmación, asi como ante otras deficientes observaciones llevadas a cabo en este lugar por el mismo autor (como calificar de glaciar a un simple nevero, próximo al E. del Lago). Pero la mayor reserva debe hacerse respecto a la posibilidad de que los hechos observados en este circo puedan permitir afirmar la viabilidad de la hipótesis del "defonçage" mantenida por Boyé.

Las solifluxiones, almohadillados, festones, polígonos, suelos estriados, etc..., no carecen de importancia, incluso por la rareza de algunos de ellos, pero no son significativos en la génesis de las grandes formas, o lo son de modo muy indirecto. Su interés es más bien eco-morfológico, en la medida que se inscriben dentro de un paisaje, que presenta en cierto modo caracteres de un "desierto frío".

\section{Los procesos torrenciales y la morfología lacustre}

En el centro del Circo, se inicia un torrente que conforma el mismo curso del Cinca. A partir del comienzo superior de las paredes de Pineta, el carácter morfofluvial se va acentuando en relación con las pendientes y aportes hídricos, cada vez más importantes.

Es prácticamente segura la acción modeladora de las aguas torrenciales en fases anteriores al glaciarismo cuaternario y, por lo tanto, en la creación del relieve preglaciar. Puede haberse reiniciado esta labor en fases interglaciares, aunque parece más discutible y matizable. Lo que es un hecho, es la absoluta prioridad que las formas glaciares y estructurales muestran hoy en día sobre las fluviales en el espacio del Circo.

Sin embargo, esta morfogénesis es actualmente funcional, junto 
a la periglaciar y glaciar: instalación de arroyos incidiendo sobre las morrenas, encajándose sobre las calizas, etc.

La acción morfológica de las aguas se manifiesta de diversos modos. En las zonas altas los torrentes forman cursos espasmódicos tras las tormentas o fuertes precipitaciones de lluvia; los pequeños arroyos de fusión de los neveros superiores puedan llegar a crear considerables cursos de carácter estacional. En la zona media, bajo las paredes, todavía no se encuentra definida la red hidrográfica y existen gran cantidad de regatos y torrentes temporales y discontinuos. Estos funcionan mientras existen neveros que les alimenten y se marcan sus cursos sobre los sedimentos glaciares o periglaciares. Por último, en las zonas más bajas del fondo del Circo, los aportes son ya suficientes para que se instale definitivamente una red clara, con caudales importantes y mucho más estables.

La red de fracturas crea una trama muy apreciable sobre paredes y escarpes, que, tras una tormenta o durante las fuertes tempestades, canaliza las aguas por buena parte de las líneas de ruptura tectónica que se ven señaladas por las blancas cascadas que salten por ellas.

Esto, sin llegar a ser tan espectacular, ocurre siempre que el aporte por precipitación o fusión es suficiente para poner en funcionamiento esta red. Las aguas son recogidas por los colectores y estos cursos no sólo evolucionan directamente por la acción erosiva de las mismas aguas, sino también indirectamente por la gelifracción posterior facilitada por la humedad.

En la zona media, bajo las paredes, las aguas superiores y las aquí aportadas por fusión, se concentran en cauces labrados sobre los sedimentos recientes. A veces se encuentran también controlados, como la deposición de los mismos sedimentos, por las directrices estructurales, a mayor o menor escala (fracturas, interestratos, pasillos, etc.).

En la zona del crestón longitudinal medio el funcionamiento es distinto, dada la carencia de aportes hídricos de las murallas superiores y el mayor dominio de la circulación subterránea. En cualquier caso no son raros, en esta zona, los pasillos subsecuentes que canalizan las aguas, pero otros, sin las pendientes necesarias y revestidos de arcillas de descalcificación, propician el estancamiento de las mismas aguas de fusión o sólo permiten una escorrentía mínima de éstas. Estos lugares son los que favorecen procesos periglaciares del estilo de los polígonos y otros tipos de ordenaciones. 
Descendiendo en altitud, los distintos aportes hídricos forman ya una definitiva red fluvial, con un curso principal -el Cinca- discurriendo tras unas planicies, que bien pudieron ser de origen lacustre. Después, el caudal sigue entre las morrenas grises menos recientes, al N., y las semiactuales al S., encajándose en un pasillo estructural. Sólo pocos metros antes de que inicie su gran salto y rápido descenso hacia el fondo de Pineta, el río se encaja en una pequeña hoz.

El arroyo del glaciar de Monte Perdido posee menos caudal è importancia. Aparece dividio en muchos brazos para irse reuniendo éstos en la morrena de ablación, por la que discurre un caudal principal que se encaja en su descenso entre dos "proas" calizas.

En cuanto a la elaboración de la cubeta lacustre donde se aloja el ibón de Tucarroya, se trata evidentemente de una sobreexcavación glaciar heredada, debida a una interacción de factores. Los glaciares del pasillo septentrional del Circo excavaron preferentemente la zona más afectada por fracturas. El cruce que la fractura de la Brecha de Tucarroya efectúa. sobre la actual depresión del valle es especialmente expresivo de este condicionamiento. Pero también la acción disolvente de las aguas ha podido tener un papel en la preparación del material al ensanchar sus planos de disyunción en un sector donde aumenta la densidad de éstos y la circulación hídrica hipogea ha podido ser alta. Pero no cabe pensar en la herencia directa de una hipotética cubeta de disolución preglaciar, ya que la excavación glaciar del Circo la hubiera borrado, ni aún menos en una forma kárstica holocena; sin embargo, es difícil encontrar la razón por la que es posible que el agua no se filtre en un roquedo potencialmente kárstico. Parece poco aceptable un factor estructural, por lo que nos inclinamos a pensar más bien en un revestimiento de arcillas en los fondos, capaz de impermeabilizar la cubeta.

La acción torrencial, ya sea fluvionival o fluviglaciar, -junto con el periglaciarismo - es el proceso que pasa a dirigir la evolución morfogenética futura del circo de Tucarroya, si no se produce un cambio en las tendencias del glaciarismo pirenáico, con posibles reavivamientos de sus actuales aparatos. 


\section{MORFOLOGIA MACIZO DE MONTE PERDIDO}

\section{REFERENCIAS BIBLIOGRAFICAS}

BARRERE, P.: "Evolution mécanique et nivation sur les versants calcaires de la haute montagne pyréneène Pirineos, 1952: 201 -211, Zaragoza.

BOYE, M.: "Importance du defonçage periglaciaire dans l'élaboration de formes glaciaires". C.R.Ac.Sc. París, 1949, págs. 723-724.

BOYE, M.: Glaciaire el periglaciaire de l'Ata Sund. NE., Groenland. París, Hermann, 1950,176 págs.

BOYE M.: "Gelivation et crioturbation dans le massif du Mont Perdu (Pyrénées Centrales)". Pirineos, 1952, págs. 5 a 29, Zaragoza.

BRUNET, G.: "Un exemple de la regresión des glaciers Pyrénéens". Pirineos, 1956, págs. $261-284$, Zaragoza.

CHOUKROUNE, P.; MARTINEZ, C.; SEGURET, M. y MATTAUER; M.: "Sur l'extension, le style et l'âge de la mise en place de la nappe de Gavarnie (Pyrénées Centrales)" C.R.Ac.Sc. París, (1968), t. 266, págs. 1360-1363, una fig.

DALLONI, M.: "Etude géologique des Pyrénées de 1'Aragón Ann. Fac. Sc. Marseille. (1910), t. 26, n. ${ }^{0} 3$, págs. 1444,56 figs.

GARCIA SAINZ, L.: "E1 glaciarismo cuaternario en el Pirineo Español" Bol. Soc. Geográfica. Madrid, 1930.

GOMEZ DE LLARENA, J.: "Algunos datos sobre el glaciar actual de Monte Perdido. Pirineos". Bol. Soc. Esp. Historia Natural tomo XXXVI. Madrid, 1936.

HERNANDEZ-PACHECO, F. y VIDAL BOX, C.: "La tectónica y la morfología del Macizo del Monte Perdido y de las zonas de cumbres inmediatas en el Pirineo Central". Zaragoza, Pirineos n. ${ }^{\circ}$ 4, 1946, págs. 69-108.

LITH, J.G.J. VAN DER.: "Geology of the Spanish part of the Gavarnie nappe (Pirénées) and its underlying sediments near Bielsa, prov. of Huesca" Thes. Univ. Utrecht, 1965.67 pág.

MALLADA, L.: Descripción física y geológica de la Provincia de Huesca. Madrid, 1878.

MARTINEZ DE PISON, E.: y ARENILLAS PARRA, M.: "El problema de 1a sobreexcavación glaciar". Estudios Geográficos, n. ${ }^{\circ}$ 145. Nov. 1976, págs. 407.434, Madrid.

MISCH, P.: "Der Bau der Mittléven Sodpyrenaen". Bertrage Zur geologie der Westlichen Mediterrangebiete. Weidmannede. Buchhandlung. Barlín, 1934.

SCHRADER, B.: Pyrénées, t. I, "Courses et ascensións"; tomo II, Sciencie et Art. "Toulouse", Privat, 1936.

SEGURET, M.: “Contribution á l'étude de la tectonique sudpyrénéenne: sur 
le style et l'importance des desplacements vers le Sud des series secondaries et tertiaires de la partie centrale des versant Sud des Pyrénées" C. R.Ac.Sc. París, 1969, t. 268, págs. 907-910.

SITTER; L.U.: "Accros sectión through the central Pyrénées" Geol. Rundschau. Tomo 45, págs. 214 a 233, 1956.

SOLER SAMPERE, M. y PUIG DE FABREGAS TOMAS, C. "Líneas generales de la Geología del Alto Aragón Occidental" Pirineos, (1970) n. . 96, pág. 5-20, un mapa geol., 2 fig, Jaca.

SOUQUET, P.: "Estructura del macizo de Monte Perdido; sus relaciones con la capa de recubrimiento de Gavarnie (Pirineos)". Not. com. del I.G.M.E. 1967, n. 03 , págs. 67-70, Madrid.

TAILLEFER, F.: "Erosión glaciaire ou défonçage periglaciaire". Rev. Geogrf. Pyr. S.O., 1952, págs. 53-57, Toulouse.

VELDE, E.J. VAN DE.: "Geology of the Ordesa overthrust mas, Spain. Pyrénées, province of Huesca”. E. Geol. 1967. vol. 23, nums. 3 y 4, págs. 163 201. 1 mapa. escala 1:120000, Madrid.

VIERS, G.: Le relief des Pyrénées occidentales et de leur piémont. Toulouse, Privat, 1960.

VIVIAN, R.: Les glaciers des Alpes occidentales. Grenoble. Ed. Allier, 1976, 513 pág. 